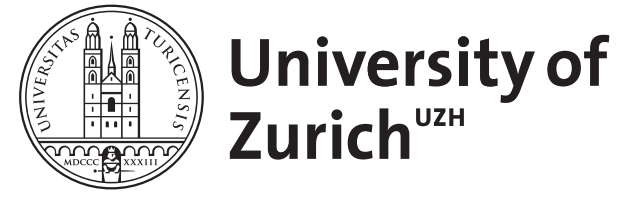
Archive

University of Zurich

University Library

Strickhofstrasse 39

CH-8057 Zurich

www.zora.uzh.ch

Year: 2020

\title{
Suitability of nitisinone for alkaptonuria
}

Häberle, Johannes

DOI: https://doi.org/10.1016/S2213-8587(20)30222-9

Posted at the Zurich Open Repository and Archive, University of Zurich

ZORA URL: https://doi.org/10.5167/uzh-197317

Journal Article

Accepted Version

Originally published at:

Häberle, Johannes (2020). Suitability of nitisinone for alkaptonuria. The Lancet. Diabetes Endocrinology, 8(9):732-733.

DOI: https://doi.org/10.1016/S2213-8587(20)30222-9 
Commentary to: Suitability of Nitisinone in Alkaptonuria 2 (SONIA 2) - An

international, multicentre, randomised, evaluator-blinded, no-treatment controlled, parallel-group study to assess the efficacy and safety of once daily nitisinone in patients with alkaptonuria after 12 months of treatment, followed by an additional 36-month treatment period

Short title: Commentary to the suitability of nitisinone in alkaptonuria

Johannes Häberle

Division of Metabolism and Children's Research Center,

University Children's Hospital Zurich,

Steinwiesstrasse 75 ,

8032 Zurich,

Switzerland

Telefon +41442667342

Telefax +41442667167

Johannes.haeberle@kispi.uzh.ch 
In many, but not all, genetic (metabolic) conditions, biochemical markers exist that are often basis for diagnosis and sometimes also for monitoring of patients. However, these markers are not always relevant for the pathogenesis of the respective conditions. For instance, in the classical urea cycle disorder citrullinemia type $1^{1}$, the name-giving metabolite citrulline, an amino acid, is largely elevated in blood and urine of affected patients but has no known role in the pathogenesis of the condition (but rather the accompanying hyperammonemia). In other conditions, the abnormal metabolite is crucial for developing disease. An illustrious example for the latter is the phenolic acid homogentisic acid, which is markedly elevated in blood and urine specifically in alkaptonuria (AKU, OMIM 203500), a disorder of phenylalanine and tyrosine degradation ${ }^{2,3}$. Importantly, this metabolite is not only found elevated but also considered central for the many complications of AKU, which mainly occur not before adulthood. Unfortunately, once developed, these complications, of which damage to ochronotic tissue resulting in premature spondyloarthritis, tendon ruptures, cardiac valve disease, and nephropathy are the most prominent, are irreversible.

Given the fact that complications in AKU occur only after many years of exposure to homogentisic acid but are then irreversible, clinical endpoints in testing efficacy of homogentisic acid-lowering therapies are prone to fail. Rather, based on the well-known natural course of the disease ${ }^{2,3}$ and on knowledge from mouse models ${ }^{4,5}$, homogentisic acid with its superior impact on the pathogenesis in this condition should not only be seen as marker for the disease but also as a suitable endpoint of clinical trials. This was done by Lakshminarayan Ranganath and colleagues from multiple European sites in a study that was long waited for in the metabolic community ${ }^{6}$. Since a blinded control study for AKU is not feasible as urinary changes with homogentisic acid-lowering therapy will unveil any blind design (urine color turning from dark/black in untreated disease condition to normal color under treatment), performing a convincing study is all the more challenging and an innovative study design is mandatory ${ }^{7}$. The current authors now were able to show a decrease in homogentisic acid 
excretion in AKU patients under nitisinone, and they additionally demonstrated statistically significant effects on some clinical outcomes ${ }^{6}$.

The paper is important for the management of AKU. So far, treatment was symptomatic and this meant the handling of inevitable ochronotic complications. Here, for a first time, not only biochemical "cure" but also clinical impact was demonstrated in a large cohort. This gives hope that the course of disease in AKU can be fundamentally changed in the future.

Nevertheless, open questions remain:

- When do we have to start treatment? After what age become the ochronotic changes irreversible and clinically relevant? For this, additional studies are needed to determine the ochronotic process during presymptomatic years and defining the optimal time point for start of treatment.

- What is the optimal dose of nitisinone? One consequence of treatment with nitisinone will be an increase of tyrosine in blood that may cause keratopathy. Patients on treatment will therefore require a phenylalanine and tyrosine reduced diet, which impacts on their quality of life. For (not only) this reason, the minimum effective dose of nitisinone should be used and this needs to be determined in future trials.

- Will all patients need to be treated? If in a patient homogentisic acid is only increased moderately, is there likewise a benefit through homogentisic acid-lowering treatment? In summary, the present study is remarkable in more than one aspect. First, AKU is an ultrarare condition affecting only 1 in 250.00 to 1.000 .000 people. To recruit 138 patients under these circumstances is simply extraordinary. Then, the study was designed as a combination of a very meaningful biochemical parameter together with clinical outcomes, and this combination of endpoints, while being unconventional, seems ideal for this very slowly progressing disease. Finally, this study gives hope that the treatment of AKU will change from a purely symptomatic therapy to prevention of complications and therefore a much improved outcome of affected 
patients. It is remarkable that this study is finally accomplished almost 22 years after the first suggestion of "multisystemic salutary effects" of nitisinone as an option to cure alkaptonuria ${ }^{8}$.

Conflicts of interest: the author declared no conflicts of interest.

\section{References}

1. Diez-Fernandez C, Rüfenacht V, Häberle J. Mutations in the Human Argininosuccinate Synthetase (ASS1) Gene, Impact on Patients, Common Changes, and Structural Considerations. Hum Mutat 2017; 38(5): 471-84.

2. Phornphutkul C, Introne WJ, Perry MB, et al. Natural history of alkaptonuria. $N$ Engl J Med 2002; 347(26): 2111-21.

3. Ranganath LR, Cox TF. Natural history of alkaptonuria revisited: analyses based on scoring systems. J Inherit Metab Dis 2011; 34(6): 1141-51.

4. Preston AJ, Keenan CM, Sutherland H, et al. Ochronotic osteoarthropathy in a mouse model of alkaptonuria, and its inhibition by nitisinone. Ann Rheum Dis 2014; 73(1): 284-9.

5. Keenan CM, Preston AJ, Sutherland H, et al. Nitisinone Arrests but Does Not Reverse Ochronosis in Alkaptonuric Mice. JIMD Rep 2015; 24: 45-50.

6. Ranganath LR, Ptychion EEP, Arnoux JP, et al. Suitability of Nitisinone in Alkaptonuria 2 (SONIA 2) - An international, multicentre, randomised, evaluator-blinded, notreatment controlled, parallel-group study to assess

the efficacy and safety of once daily nitisinone in patients with alkaptonuria after 12 months of treatment, followed by an additional 36-month treatment period. The Lancet Diabetes \& Endocrinology 2020; in press.

7. Cox TM. Alkaptonuria: treasure your exceptions. J Inherit Metab Dis 2011; 34(6): 1113-4.

8. Anikster Y, Nyhan WL, Gahl WA. NTBC and alkaptonuria. Am J Hum Genet 1998; 63(3): 920-1. 